\title{
Measuring the Benefits of Mass Vaccination Programs in the United States
}

\author{
Hector Magno ${ }^{1}$ and Beatrice Golomb ${ }^{2, *}$ \\ 1 Independent Computer Scientist, Orange County, CA 92677, USA; hmagno@protonmail.com \\ 2 Department of Medicine, UC San Diego School of Medicine, La Jolla, CA 92093, USA \\ * Correspondence: bgolomb@health.ucsd.edu; Tel.: +858-558-4950
}

Received: 26 August 2020; Accepted: 22 September 2020; Published: 29 September 2020

\begin{abstract}
Since the late 1940s, mass vaccination programs in the USA have contributed to the significantly reduced morbidity and mortality of infectious diseases. To assist the evaluation of the benefits of mass vaccination programs, the number of individuals who would have suffered death or permanent disability in the USA in 2014, had mass vaccination never been implemented, was estimated for measles, mumps, rubella, tetanus, diphtheria, pertussis, polio, Haemophilus influenzae type $b(\mathrm{Hib})$, hepatitis B, varicella, and human papillomavirus (HPV). The estimates accounted for mortality and morbidity trends observed for these infections prior to mass vaccination and the impact of advances in standard of living and health care. The estimates also considered populations with and without known factors leading to an elevated risk of permanent injury from infection. Mass vaccination prevented an estimated 20 million infections and 12,000 deaths and permanent disabilities in 2014, including 10,800 deaths and permanent disabilities in persons at elevated risk. Though 9000 of the estimated prevented deaths were from liver cirrhosis and cancer, mass vaccination programs have not, at this point, shown empirical impacts on the prevalence of those conditions. Future studies can refine these estimates, assess the impact of adjusting estimation assumptions, and consider additional risk factors that lead to heightened risk of permanent harm from infection.
\end{abstract}

Keywords: vaccination; disease; mortality; disability; risk

\section{Introduction}

To measure the benefit of a mass vaccination program targeting an infectious disease, it is useful to assess what the risk of death or permanent injury would be from the disease in the absence of the mass vaccination program. There is an abundance of medical literature detailing the risks associated with infectious diseases; however, the information is scattered through dozens of sources that are often lengthy and consider only a narrow scope of the risks involved. For example, some sources describe the symptoms of a disease without specifying how many patients fully recover [1]; other sources describe the number of deaths from an infection without addressing permanent disability in survivors [2,3]. Moreover, some sources do not account for the pre-vaccine rates of decline in mortality for some infectious diseases [3-5]. We tried to address these challenges in our estimates.

\section{Materials and Methods}

We calculated rates of deaths and disabilities from infectious diseases, had mass vaccination never been implemented, using data principally from reports of the Centers for Disease Control and Prevention (CDC), complemented by reports from other federal entities such as the US Bureau of the Census and the US Public Health Service. We relied on data recorded in scientific journals (e.g., The Journal of the American Medical Association, Pediatrics, The Journal of Infectious Diseases, The New England Journal of Medicine, and The Journal of Clinical Oncology) in cases when data from 
government sources were unavailable or incomplete. For example, although the impact of risk factors for many diseases is considered in CDC data, there are instances when measurements of risk factor relationships to outcomes are not provided. Other examples of information that is not always available in government records include estimates of the number of unreported cases and of permanent disability from certain diseases. In addition, when US data for measurements were unavailable, we relied on data from other developed countries.

Because we researched and gathered data for our estimates in 2016, when the latest available CDC mortality data were from 2014, we projected our estimates to 2014. When calculating the rates of deaths and disabilities corresponding to an infectious disease, we considered the trend in deaths and disabilities during a range of years just prior to the licensing of a vaccine or at the start of a nationwide mass vaccination program targeting the disease. This range of years is referred to as the "reference years." The duration of each range was chosen by using the number of years between relative peaks of incidence of each disease.

Though $<100 \%$ of the population is vaccinated and vaccines are $<100 \%$ effective, mass vaccination programs have contributed to the significantly reduced morbidity and mortality of infectious diseases. There were fewer than two dozen deaths from diphtheria, tetanus, pertussis, polio, measles, mumps, rubella, Haemophilus influenzae type b (Hib), or varicella in 2014. Therefore, we counted all estimated cases projected to 2014 in the absence of mass vaccination as preventable.

We employed certain assumptions in our estimates. For example, we presumed that each vaccine neither reduced nor enhanced vulnerability to the incidence or outcomes of diseases that were not targeted by the vaccine. Throughout our text, we strive to be explicit whenever an assumption was made.

The rates of deaths or disabilities corresponding to various infectious diseases were computed using a denominator of 307 million individuals $<80$ years of age in 2014 . We chose that age group because the life expectancy in 2014 was 79 years. In instances where age-specific counts of cases of infection were not available, case counts from the entire US population (319 million) were used. Moreover, we considered the broader population $<80$ years of age rather than the population of children, both because mass vaccination programs are intended to provide lifetime immunity to infection and because protecting against infection averts late complications of infection. For example, the polio mass vaccination program affected not only permanent injury from polio in children but also permanent injury from post-polio syndrome in adults.

To simplify and reduce the length of the report, we omitted analyses of certain mass vaccination programs that had marginal or unclear impacts on mortality. We excluded rotavirus and hepatitis A because each of those infections caused fewer than 100 annual pre-vaccine deaths [2,6]. We excluded influenza because its mass vaccination program has not made a clear impact on the trend of its pre-vaccine mortality rate [7-9]. Though there is an effective vaccine targeting meningococcal disease, the trend in the mortality rate for that infection after the introduction of its mass vaccination program has resembled the pre-vaccine trend [10]. Consequently, we excluded meningococcal disease because the matching mortality rate trends suggested that fewer than 100 annual deaths were prevented. We excluded pneumococcal disease for a similar reason. The CDC's estimated decline in pneumococcal disease mortality after the introduction of the mass vaccination program matched the rate of decline in mortality from all pneumonia, including pneumonia caused by pathogens that are not targeted by the vaccine. From 2000 to 2009, the CDC estimated that pneumococcal disease mortality declined from 2.3 per 100,000 population to 1.6 , a $30 \%$ decline [11,12]. During the same time period, the mortality rate of pneumonia from all causes declined from 22.6 to 16.6 , a $27 \%$ decline [13]. The similar rates of decline suggested that the mass vaccination program prevented fewer than 100 deaths among individuals $<80$ years of age.

When accounting for risk factors leading to an elevated risk of permanent injury from an infection, we included only those factors that were observed in a high fraction of cases of permanent injury from the infection. To simplify the report, risk factors that were present in a small fraction of such cases 
were excluded if accounting for those factors resulted in a rate of permanent injury that lay within the $95 \%$ confidence interval of the rate computed without those factors (i.e., did not make a statistically significant impact on the rate).

\section{Results}

It was estimated that 20 million infections and 12,000 deaths and permanent disabilities may have occurred in 2014 in the absence of mass vaccination, with 10,800 deaths and disabilities among individuals who have conditions or behaviors that would put them at higher risk of such outcomes and 1200 deaths and disabilities among persons without those conditions or behaviors. Tables 1 and 2 show the aggregated results for the infectious diseases examined in this report.

The following is a discussion of each disease. Recall that the "reference years" refer to the time period before the introduction of the corresponding mass vaccination program. Using data recorded during these years, we derived estimates of the expected number of deaths and permanent disabilities from each disease had mass vaccination not been introduced.

\subsection{Measles}

During the reference years of 1959-1962, before the introduction of mass vaccination, there were four million annual measles cases (equal in size to the birth cohort; Table S1A) that resulted in 402 deaths [2] mostly among the population $<10$ years of age [14]. Because the birth cohorts in the 1960s and in 2014 were the same size and the number of susceptible children was also the same [15,16], we estimated that these values would remain unchanged in the absence of mass vaccination. We also estimated 106 additional cases of measles that resulted in residual neurologic damage from complications of measles including measles encephalitis and subacute sclerosing panencephalitis (Table S1B).

Individuals with low levels of vitamin A are significantly more likely to suffer death or permanent disability from measles [17,18]; $92 \%$ of the most severe measles cases have had low levels of vitamin A (Table S1C) [19]. Therefore, we calculated 467 (=92\% of 508) measles deaths and permanent disabilities at elevated risk.

Though the pre-vaccine measles mortality rate declined from 14.1 to 0.2 per 100,000 people (Figure S1), the measles fatalities recorded in the 1980s and 1990s suggested that the pre-vaccine decline may not have continued as rapidly in the absence of mass vaccination [17]. Consequently, we assumed that the measles mortality rate would have remained unchanged from the reference years.

\subsection{Mumps}

During the reference years of 1963-1966, before the introduction of mass vaccination, there were four million annual mumps cases (equal in size to the birth cohort; Table S2A) that resulted in 43 deaths [2], mostly among the population <30 years of age [20]. Because the birth cohorts in the 1960s and in 2014 were the same size and the number of susceptible children was also the same [15,16], we estimated that these values would remain unchanged in the absence of mass vaccination. We also estimated 11 additional cases of mumps resulting in permanent impaired hearing and 7 additional cases of mumps resulting in permanent impaired fertility (Table S2B). 
Table 1. Estimated rates of death and permanent disability from various infectious diseases in the USA in the absence of mass vaccination among normal and high risk individuals $<80$ years of age, 2014

\begin{tabular}{|c|c|c|c|c|c|c|c|c|c|}
\hline \multirow[t]{2}{*}{ Infection } & \multirow{2}{*}{$\begin{array}{c}\text { Range of } \\
\text { Reference Years } \\
\text { Used for } \\
\text { Estimates }\end{array}$} & \multirow{2}{*}{$\begin{array}{l}\text { Number of } \\
\text { Cases } \\
\text { (Morbidity) } \\
\text { (A) }\end{array}$} & \multicolumn{2}{|c|}{$\begin{array}{l}\text { Population in } 100,000 \mathrm{~s} \\
\text { (B) }\end{array}$} & \multicolumn{2}{|c|}{$\begin{array}{l}\text { Estimated Number of Cases of } \\
\text { Death and Permanent Disability } \\
\text { (C) }\end{array}$} & \multirow{2}{*}{ 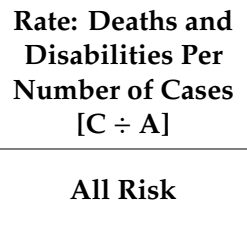 } & \multicolumn{2}{|c|}{$\begin{array}{c}\text { Rate: Deaths and Disabilities Per } \\
100,000 \text { Population } \\
{[C \div \text { B] }(95 \% \text { C.I. })}\end{array}$} \\
\hline & & & $\begin{array}{c}\text { Normal } \\
\text { Risk }^{a}\end{array}$ & $\begin{array}{l}\text { High } \\
\text { Risk }^{\text {a }}\end{array}$ & $\begin{array}{c}\text { Normal } \\
\text { Risk }^{a}\end{array}$ & $\begin{array}{l}\text { High } \\
\text { Risk }^{a}\end{array}$ & & $\begin{array}{c}\text { Normal } \\
\text { Risk }^{a}\end{array}$ & $\begin{array}{l}\text { High } \\
\text { Risk }^{\text {a }}\end{array}$ \\
\hline Measles & 1959-1962 & $4,000,000$ & 2920 & 150 & 41 & 467 & $0.01 \%$ & $0.014(0.010-0.018)$ & 3.11 (2.83 to 3.40 \\
\hline Mumps & 1963-1966 & $4,000,000$ & 3070 & 0 & 61 & 0 & $0.002 \%$ & $0.020(0.015-0.025)$ & 0 \\
\hline Rubella & 1960-1968 & $4,000,000$ & 3000 & 70 & 19 & 140 & $0.004 \%$ & $0.006(0.003-0.009)$ & $2.00(1.67-2.33)$ \\
\hline Tetanus & 1943-1945 & 1800 & 3070 & 0 & 113 & 0 & $6.3 \%$ & $0.037(0.029-0.043)$ & 0 \\
\hline Diphtheria & 1879-1945 & 560 & 3070 & 0 & 28 & 0 & $5 \%$ & $0.009(0.006-0.012)$ & 0 \\
\hline Pertussis & 1943-1945 & $1,300,000$ & 3070 & 0 & 123 & 0 & $0.009 \%$ & $0.040(0.033-0.047)$ & 0 \\
\hline Polio & 1935-1954 & 72,500 & 2480 & 590 & 353 & 1149 & $2.1 \%$ & $0.142(0.127-0.157)$ & $1.95(1.83-2.06)$ \\
\hline Hib & 1980-1984 & 2800 & 1440 & 1630 & 66 & 208 & $9.8 \%$ & $0.046(0.035-0.057)$ & $0.13(0.11-0.14)$ \\
\hline Hepatitis B & 1988-1990 & 190,000 & 2610 & 460 & 106 & 3034 & $1.7 \%$ & $0.041(0.033-0.048)$ & $6.60(6.36-6.83)$ \\
\hline Varicella & 1991-1994 & $4,000,000$ & 3070 & 0 & 101 & 0 & $0.003 \%$ & $0.033(0.026-0.039)$ & 0 \\
\hline HPV & 2011-2014 & $2,800,000$ & 1750 & 1320 & 198 & 5711 & $0.2 \%$ & $0.113(0.097-0.129)$ & $4.33(4.22-4.44)$ \\
\hline
\end{tabular}

a "High risk" refers to individuals with specified factors linked to an elevated risk of permanent injury from the infection. "Normal risk" refers to individuals without those specific known factors and also refers to individuals with risk factors that were not identified or were excluded in our analysis. High risk factors, by infection, include: measles-insufficient vitamin A; rubella—woman who had not contracted rubella before pregnancy; polio—absence of tonsils and not resting after the onset of significant symptoms; Haemophilus influenzae type b (Hib) - breastfed for < 13 weeks; hepatitis B - infant of an infected mother, dwelling with an infected individual, sex with an infected partner, sex with multiple partners, men having sex with men, injection-drug use, and dwelling in a community with an unusually large group of infected individuals; and human papillomavirus (HPV)—smoking, women not screened every 3 years, and men with $\geq 6$ oral sex partners in their lifetime. 
Table 2. Age groups that comprised the greatest proportion of deaths and permanent disabilities from various infectious diseases in the USA in the absence of mass vaccination.

\begin{tabular}{ccc}
\hline Infection & Age Group & $\begin{array}{c}\text { Proportion of Deaths and } \\
\text { Permanent Disabilities }\end{array}$ \\
\hline Measles & $<10$ & $91 \%$ \\
\hline Mumps & $<30$ & $59 \%$ \\
\hline Rubella & in utero & $88 \%$ \\
\hline Tetanus & $<20$ & $53 \%$ \\
\hline Diphtheria & $1-9$ & $78 \%$ \\
\hline Pertussis & $<1$ & $71 \%$ \\
\hline Polio & $<15$ & $54 \%$ \\
\hline Hib & $<5$ & $99 \%$ \\
\hline Hepatitis B & $\geq 50$ & $79 \%$ \\
\hline Varicella & $\geq 20$ & $54 \%$ \\
\hline HPV & $\geq 50$ & $86 \%$ \\
\hline
\end{tabular}

\subsection{Rubella (German Measles)}

During the reference years of 1960-1968, before the introduction of mass vaccination, there were four million annual rubella cases (equal in size to the birth cohort; Table S3A) that resulted in 19 deaths [2]. CDC analyses of rubella have shown that permanent disability in rubella survivors was very rare [21]. However, mass vaccination was adopted because of congenital rubella syndrome (CRS) [21], which posed a threat to those infants whose mothers were infected by rubella during the first two trimesters of pregnancy. Therefore, we additionally considered the number of babies that contracted rubella in utero.

Using the government tracking of cases of rubella and CRS, we estimated that during the nine reference years, there were 1484 cases of CRS or 165 cases annually (Table S3B). Of those cases, we calculated $140(=85 \%$ of 165$)$ that resulted in death or permanent disability [21].

Because the birth cohorts in the 1960s and in 2014 were the same size and the number of susceptible children was also the same $[15,16]$, we estimated that these values would remain unchanged in the absence of mass vaccination. Furthermore, most women in the 1960s contracted rubella before childbearing age [22], and the typical childbearing age of women in 2014 was greater than it was in the 1960s. Therefore, the estimated 165 cases of CRS for 2014 may be an overestimation.

\subsection{Tetanus}

Using the government tracking of cases of tetanus, we calculated that, during the reference years of 1943-1945, before the introduction of mass vaccination, one case of tetanus occurred in every 180,000 people (Table S4A). To estimate the number of tetanus cases for 2014 in the absence of mass vaccination, we multiplied the pre-vaccine incidence ratio of 1 in 180,000 by 2014's population ( 319 million) to obtain 1800 cases of tetanus.

CDC analyses of tetanus have shown that permanent disability in tetanus survivors was very rare [23]. As for deaths from tetanus, we used the most recent case fatality rates recorded among unvaccinated populations to account for significant improvements in health care and other factors influencing disease outcomes since the 1940s. From 2001 to 2008, we calculated a case fatality rate of $6.3 \%$ among unvaccinated individuals $<80$ years of age (Table S4B). We multiplied the estimated 1800 cases of tetanus for 2014 by the case fatality rate of $6.3 \%$ to obtain 113 fatal cases. 
The pre-vaccine decline in the tetanus mortality rate from 2.4 to 0.5 tetanus deaths per 100,000 people (Figure S2) provided additional support for the projected decline in annual tetanus deaths from 626 in the 1940s (Table S4A) to 113 in 2014.

\subsection{Diphtheria}

During the reference years of 1879-1945, before the introduction of the national mass vaccination program, there was an exponential decline in diphtheria morbidity and mortality (Figure S3) [24-26]. This decline predated the introduction of antitoxin in the late 1890s, the introduction of toxin-antitoxin in the 1920s, and the gradual introduction of toxoid in the 1930s [27,28]. Furthermore, none of those events significantly altered the decline, suggesting that non-vaccine factors played important roles [27,28]. Laboratory testing revealed the protective effects of vitamin C [29], iron [30], and vitamin B3 [31] against diphtheria toxin. Studies also revealed that crowding and low levels of hygiene were associated with high incidences of diphtheria [32].

Because significant improvements in nutrition, sanitation, living conditions, and access to health care continued after the reference years, we estimated that the 65-year decline would have continued in the absence of the national mass vaccination program of the late 1940s, and we calculated 28 diphtheria deaths for 2014 (Table S5). Because the prevalence and severity of diphtheria risk factors required to put an individual at elevated risk have not been measured, we did not attempt to segregate cases at high risk from our results.

CDC analyses of diphtheria have shown that permanent disability in diphtheria survivors was very rare [28]. Additionally, given a case fatality rate of 5\% for diphtheria [28], we estimated 560 (=28/5\%) diphtheria cases for 2014 .

\subsection{Pertussis (Whooping Cough)}

Using the government tracking of cases of pertussis during the reference years of 1943-1945, before the introduction of mass vaccination, we calculated 235,000 reported pertussis cases out of a total 1.3 million cases for 2014 in the absence of mass vaccination (Table S6A).

CDC analyses of pertussis have shown that permanent disability in pertussis survivors was very rare [33]. As for deaths from pertussis, we used the most recent case fatality rates recorded among unvaccinated populations to account for significant improvements in health care and other factors influencing disease outcomes since the 1940s. From 2012 to 2014, we calculated a reported case fatality rate of $0.7 \%$ among unvaccinated infants $<3$ months of age (Table S6B). We estimated 4500 reported pertussis cases for 2014 in that age group (Table S6C) and multiplied by the reported case fatality rate of $0.7 \%$ to obtain 32 fatal cases. Because infants $<3$ months of age comprised $26 \%$ of all pertussis deaths during the reference years (Table S6D), we calculated 123 (=32/26\%) pertussis deaths among individuals of all ages.

The pre-vaccine decline in the pertussis mortality rate from 16.1 to 1.3 pertussis deaths per 100,000 people (Figure S4) and the mortality rate of one in eight million recorded in Sweden in the absence of mass vaccination in the 1980s [34] provided additional support for the projected decline in annual pertussis deaths from 2300 in the 1940s (Table S6D) to 123 in 2014.

\subsection{Polio}

During the reference years of 1935-1954, before the introduction of mass vaccination, there were an estimated 7260 annual cases of paralytic poliomyelitis (Table S7A), of which 1136 resulted in death or permanent disability (Table S7B,C). Since $95 \%$ of all polio infections were unnoticed or asymptomatic, and less than $1 \%$ of cases were paralytic [35], we estimated at total of $36,000(\approx 5 \%$ of $(7260 / 1 \%))$ noticeable annual cases of polio. To estimate the number of noticeable cases of polio for 2014 in the absence of mass vaccination, we multiplied the pre-vaccine incidence ratio of 1 in $4400(\approx 36,000 / 160$ million) by the 2014 population (319 million) to obtain 72,500 cases. 
Individuals without tonsils or who do not rest after the onset of significant symptoms are more likely to suffer permanent disability or death from paralytic poliomyelitis [36,37]. We calculated 1149 permanent disabilities and deaths among individuals at elevated risk and 353 among individuals at normal risk for 2014 in the absence of mass vaccination (Table S7D-H).

\subsection{Hib (Haemophilus Influenzae Type b)}

During the reference years of 1980-1984, before the introduction of mass vaccination, most children acquired immunity to Hib by five years of age through asymptomatic infection. In this report, we only considered identifiable cases of Hib-invasive Hib cases [38].

Using invasive $H$. influenzae data from the reference years and the government tracking of cases of invasive $H$. influenzae, we estimated an annual total of 3400 cases of invasive Hib for 1994-2000 in the absence of mass vaccination (Table S8A,B). Of those cases, we calculated 330 resulting in death or permanent disability from meningitis, bacteremia, or epiglottitis (Table S8C).

Children who were breastfed exclusively for $\geq 13$ weeks were 2.8 times less likely to contract invasive Hib (Table S8D). On this basis, we used our estimates of invasive Hib incidence and permanent injury for 1994-2000 to calculate estimates for 2014 in the absence of mass vaccination: 66 cases of permanent injury among children breastfed exclusively for $\geq 13$ weeks and 208 cases of permanent injury among children breasted for $<13$ weeks (Table S8E,F).

To estimate the number of all cases of invasive Hib for 2014, we divided the 274 cases of death and permanent disability by the percentage of Hib cases that resulted in such outcomes-9.8\% $(=[60 \%$ $\times(11 \%+5 \%)]+[15 \% \times 1 \%] ;$ Table S8C) — to obtain 2800 cases.

\subsection{Hepatitis $B$}

Using the government tracking of cases of hepatitis B during the reference years of 1988-1990, before the introduction of mass vaccination, we calculated 190,000 cases for 2014 in the absence of mass vaccination (Table S9A), including 300 cases of fatal fulminant hepatitis-nearly all of which occurred in adults and adolescents (Table S9B).

CDC analyses of hepatitis B have shown that permanent disability in hepatitis B survivors is very rare [39]. However, a portion of hepatitis B survivors can develop a chronic infection that can lead to fatal cirrhosis or liver cancer later in life, and $85 \%$ of those deaths occur in individuals $<80$ years of age [40]. Using government chronic hepatitis B data, we estimated 1100 infections in adults and adolescents and 1740 infections in children resulting in chronic infection that led to death before age 80 for 2014 (Table S9C).

Individuals at high risk of exposure are more likely to contract hepatitis B (Table 3). Of the estimated 1400 deaths among adults and adolescents for 2014 in the absence of mass vaccination (300 from fulminant hepatitis + 1100 from chronic infection), we calculated 1300 deaths among individuals at elevated risk (Table S9D). Of the estimated 1740 deaths from infections in childhood, we calculated 1734 among children at elevated risk (Table S9E). Combining these totals resulted in $3034(=1300+$ $1734)$ hepatitis B-related deaths among individuals at elevated risk and $106(=100+6)$ deaths among individuals at normal risk.

Table 3. Risk factors for elevated exposure to hepatitis B.

\begin{tabular}{cc}
\hline Age Group & Risk Factors \\
\hline Children & $\begin{array}{c}\text { Being born to a chronically infected mother, living with a chronically infected } \\
\text { individual, and dwelling in a community that has a large number of infected } \\
\text { individuals }\end{array}$ \\
\hline Adults and adolescents & $\begin{array}{c}\text { Having multiple sex partners, men having sex with men, injection-drug use, and } \\
\text { dwelling in a community that has a large number of infected individuals }\end{array}$ \\
\hline
\end{tabular}




\subsection{Varicella (Chicken Pox)}

During the reference years of 1991-1994, before the introduction of mass vaccination, there were four million annual varicella cases (equal in size to the birth cohort; Table S10) that resulted in 101 deaths [2], mostly among the population $\geq 20$ years of age [41]. Because the birth cohorts in the 1990s and in 2014 were the same size and the number of susceptible adults was also the same [16,42], we estimated that this value would remain unchanged in the absence of mass vaccination.

CDC analyses of varicella have shown that permanent disability in varicella survivors is very rare [43]. Though zoster (shingles) can occur later in life in individuals infected with varicella, death or permanent disability from zoster is also very rare [44]. Thus, we estimated no cases of varicella-related permanent disability for 2014 .

\subsection{HPV (Human Papillomavirus)}

The CDC has estimated that there are 14 million annual cases of HPV of all types [45]. Of those 14 million cases, 20\% (2.8 million) are targeted by vaccines [46]. Most HPV infections are unnoticed or asymptomatic. When there are symptoms from an HPV infection, such as genital warts, they very rarely cause death or permanent disability [45]. However, a small proportion of individuals infected with HPV can become persistently infected, and this condition can lead to various kinds of cancers later in life $[45,47]$. The first HPV vaccine was licensed in 2006, and the vaccination program targeted teenagers. Since HPV-attributable cancers rarely affect individuals $<30$ years of age, it will take at least another decade before it is possible for mass vaccination to have a measurable effect on the incidence of those cancers. Here, we consider HPV-attributable cancer statistics during the reference years of 2011-2014.

Table 4 contains a list of HPV-attributable cancers and factors that lead to an elevated risk of dying from them. Among women $<80$ years of age, we estimated 132 fatal HPV-attributable cancers occurring in women at normal risk and 5340 fatal cancers occurring in women at elevated risk (Table S11A). Among men <80 years of age, we estimated 66 fatal HPV-attributable cancers occurring in men at normal risk and 371 fatal cancers occurring in men at elevated risk (Table S11B). Combining these totals resulted in $198(=132+66)$ HPV-related deaths among individuals at normal risk and 5711 $(=5340+371)$ deaths among individuals at elevated risk.

Table 4. Types of HPV-attributable cancer and factors that elevate the risk of dying from them.

\begin{tabular}{ccc}
\hline Gender & HPV-Attributable Cancers & Risk Factors \\
\hline Female & $\begin{array}{c}\text { Cervix, vagina, vulva, anus, rectum, and } \\
\text { oropharynx }\end{array}$ & $\begin{array}{c}\text { No Pap or HPV screening every three } \\
\text { years and smoking }\end{array}$ \\
\hline Male & Oropharynx, penis, anus, and rectum & $\begin{array}{c}\text { Smoking and having six or more oral } \\
\text { sex partners in a lifetime }\end{array}$ \\
\hline
\end{tabular}

\section{Discussion}

Based on population data for 2014, it was estimated that mass vaccination programs against measles, mumps, rubella, tetanus, diphtheria, pertussis, polio, Hib, hepatitis B, varicella, and HPV could prevent 20 million infections and 12,000 deaths and permanent disabilities annually.

Individuals who have conditions or behaviors that would put them at higher risk of permanent injury from infectious diseases (e.g., insufficient vitamin A, absence of tonsils, breastfed $<13$ weeks, injection-drug use, and smoking) were found to comprise $90 \%(\approx 10,800 / 12,000)$ of all the estimated cases of prevented death and permanent disability, with the remaining 1200 cases in persons at normal risk (or with risk factors excluded from this report). It is possible that the high risk conditions described in this report might expose individuals to permanent harm from other causes. More research in this arena would be useful. 
Pre-vaccine declines in mortality rates recorded for measles, tetanus, diphtheria, and pertussis were not unique to those infections. In the early 20th century, significant declines in mortality rates were recorded for numerous infectious diseases that were not targeted by mass vaccination programs, such as those for tuberculosis, syphilis, typhoid fever, and dysentery [48]. The human immune system is evidently remarkably efficient when coupled with treatments for severe cases of diseases, such as antibiotics and when not hampered by factors like poor nutrition, poor sanitation, or limited access to health care.

Mass vaccination programs are best known for preventing deaths and permanent disabilities that occur a relatively short time after infection. However, $75 \%(\approx 9000 / 12,000)$ of the estimated cases of death and permanent disability prevented in this report would be from conditions occurring much later in life-liver cirrhosis and cancer. Though hepatitis B and HPV are the causes of these conditions, the hepatitis B and HPV mass vaccination programs have not, at this point, shown empirical impacts on the prevalence of liver cirrhosis and cancer. In spite of the significant reduction in acute cases of hepatitis B, the prevalence of chronic hepatitis B has remained practically unchanged since 1976 [49]. As for the HPV vaccine, although the prevention of HPV infections that are necessary for the potential development of cancer has been observed [45], cancer protection has not yet been empirically documented and uncertainties remain. Among these, a minimum protective antibody titer has not been determined [45], and the duration of antibody response has only been measured for eight-to-nine years [50]. Since most HPV-attributable cancers occur in the population $>50$ years of age, it may be that to most successfully prevent HPV-attributable cancer, either an HPV vaccine needs to provide lifetime immunity or booster doses need to be introduced into the mass vaccination program.

This report had other limitations. The accuracy of our estimates depended on the quality of the available evidence concerning the risks and effects of the diseases, which could have been imperfect. In many instances, we projected to 2014 from statistics recorded decades earlier. The accuracy of such projections could have been affected by changes in the organisms targeted by vaccines, changes in host resilience, or changes in health care practices deviating from pre-vaccine trends, among other factors. In addition, inaccuracies can propagate from one estimate to another when an estimate is used to derive the other. For example, the case fatality rate of a disease is sometimes used to estimate the total number of cases of that disease based on its estimated number of fatalities. Another limitation was related to the potential aggregate impact of risk factors that were not considered in generating our estimates, either because they are (individually) less commonly implicated or are undiscovered or inadequately studied. The comprehensive consideration of such factors might shift more of the vaccine-averted deaths and disability to the high-risk category. The same limitations might apply to the long term effects of some of the diseases. It is also possible that relevant information was missed in our literature review. We sought to convey the challenges surrounding some of the available data in the discussion of each disease and have tried to be explicit about assumptions made. We explained our choices in relation to the application of pre-vaccine trends and in gauging expected disease outcomes as a function of whether an individual is at higher risk. Furthermore, this study only estimated the number of deaths and permanent disabilities prevented by mass vaccination programs. It did not consider similar outcomes that may be caused by these programs.

Despite these limitations, we believe this report employed the best processes among the available data and studies for estimating the numbers of deaths and permanent disabilities that would have occurred (here estimated for 2014) in the absence of mass vaccination programs. Though other studies have presented estimates of the benefits of mass vaccination programs, they have not accounted for disease risk factors, cases of nonfatal permanent disability, pre-vaccine trends in mortality, post-vaccine improvements in factors tied to disease outcomes resulting in improved case fatality rates in unvaccinated populations (such as improved nutrition, sanitation, hygiene, indoor temperature control, health care, and the treatment of disease), and adjustments of pre-vaccine estimates using data recorded after vaccine licensure [3-5]. Furthermore, some studies have not provided an explanation 
for the data used as the basis of their estimates [4,5]. We have tried to rectify these omissions in the present report.

\section{Conclusions}

Despite the decline in mortality rates of infectious diseases recorded since the late 19th century, the data in this report indicate that mass vaccination programs may still have prevented 20 million infections and 12,000 deaths and permanent disabilities in 2014. In addition, mass vaccination programs have reduced the burden on health services, hospitals, intensive care, and the economy caused by the diseases they target. Put another way, measuring the number of deaths and cases of permanent disability prevented by mass vaccination programs is not the only way to measure the benefit of those programs, as those benefits can also be measured by other outcomes such as hospitalizations or the economic burden associated with a disease. However, those outcomes may be more greatly influenced by a range of factors beyond the impact of the vaccine and the disease, such as shifts in approaches to and costs of hospitalization over time. Nonetheless, because such outcomes are generally a function of the morbidity and mortality of the disease, the data in this report might also be useful in generating estimates for those outcomes.

We believe this report provides a useful reference for the effect of mass vaccination programs on the most serious complications of the diseases they target. Future studies can seek to further refine these estimates, use these estimates in risk-benefit analyses, and assess how adjusting assumptions influences effect estimates.

Supplementary Materials: The following are available online at http://www.mdpi.com/2076-393X/8/4/561/s1: Table S1: Basis for figures concerning measles; A. Number of measles cases, 1959-1962; B. Estimated number of permanent disabilities from measles for 2014 in the absence of mass vaccination; and C. Percentage of severe measles cases that have low levels of vitamin A. Figure S1: Decline in measles mortality, 1900-1960. Table S2: Basis for figures concerning mumps; A. Number of mumps cases, 1963-1966; and B. Estimated number of permanent disabilities from mumps for 2014 in the absence of mass vaccination. Table S3: Basis for figures concerning rubella; A. Number of rubella cases, 1960-1968; and B. Estimated number of cases of CRS, 1960-1968. Table S4: Basis for figures concerning tetanus; A. Estimated incidence of tetanus, 1943-1945; and B. Tetanus case fatality rate among unvaccinated individuals $<80$ years of age, 2000-2008. Figure S2: Decline in tetanus mortality, 1900-1945. Table S5: Basis for figures concerning diphtheria. Figure S3: Decline in diphtheria mortality, 1879-1945. Table S6: Basis for figures concerning pertussis; A. Estimated number of cases of pertussis for 2014 in the absence of mass vaccination; B. Pertussis reported case fatality rate among unvaccinated infants $<3$ months of age, 2012-2014; C. Estimated number of cases of pertussis among infants $<3$ months of age for 2014 in the absence of mass vaccination; and D. Number of pertussis deaths and percentage that occurred among infants $<3$ months of age, 1943-1945. Figure S4: Decline in pertussis mortality, 1900-1945. Table S7: Basis for figures concerning polio; A. Estimated number of cases of paralytic poliomyelitis, 1935-1954; B. Estimated number of poliomyelitis cases resulting in permanent disability or death, 1935-1954; C. Estimated case fatality rate of paralytic poliomyelitis, 1935-1954; D. Estimated number of poliomyelitis cases resulting in death or permanent disability for 2014 in the absence of mass vaccination; E. Estimated risk of permanent disability or death from poliomyelitis in children $<10$ years of age based on tonsillectomy and rest status, 1935-1954; F. Estimated risk of permanent disability or death from poliomyelitis in individuals 10-39 years of age based on tonsillectomy and rest status, 1935-1954; G. Percentage of population that had no tonsils, 1935-1954; and H. Percentage of poliomyelitis cases that rested after significant onset of symptoms by type of paralysis and severity, 1935-1954. Table S8: Basis for figures concerning Haemophilus influenzae type b, A. Estimated number of cases of invasive Hib in the absence of mass vaccination, 1994-2000; B. Invasive H. influenzae tracking in children <5 years of age, 1994-2000; C. Estimated number of cases of invasive Hib resulting in death or permanent disability in the absence of mass vaccination, 1994-2000; D. Protective effect of breastfeeding against invasive Hib measured in 1997 Swedish study of children $<6$ years of age; E. Estimated number of cases of invasive Hib resulting in death or permanent disability for 2014 in the absence of mass vaccination based on breastfeeding status; and F. Estimated risk of invasive Hib based on breastfeeding status in the absence of mass vaccination, 1994-2000. Table S9: Basis for figures concerning hepatitis B; A. Estimated number of cases of hepatitis B for 2014 in the absence of mass vaccination; B. Estimated number of cases of fatal fulminant hepatitis B for 2014 in the absence of mass vaccination; C. Estimated number of hepatitis B cases leading to death from chronic infection before age 80 for 2014 in the absence of mass vaccination among various age groups; D. Estimated number of hepatitis B cases leading to death before age 80 for 2014 in the absence of mass vaccination among adults and adolescents at high risk of exposure; and E. Estimated number of hepatitis B cases leading to death before age 80 for 2014 in the absence of mass vaccination among children at high risk of exposure. Table S10: Basis for figures concerning varicella. Table S11: Basis for figures concerning human papillomavirus; A. Estimated number of HPV cases leading to death before age 80 for 2014 among women; and B. Estimated number of HPV cases leading to death before age 80 for 2014 among men. 
Author Contributions: Conceptualization, H.M.; methodology, H.M.; validation, B.G.; formal analysis, H.M.; investigation, H.M. and B.G.; data curation, H.M.; writing-original draft preparation, H.M.; writing-review and editing, B.G.; supervision, B.G.; project administration, H.M. and B.G. All authors have read and agreed to the published version of the manuscript.

Funding: This research received no external funding.

Conflicts of Interest: The authors declare no conflict of interest.

\section{References}

1. Centers for Disease Control and Prevention. Available online: https://www.cdc.gov/vaccines/hcp/vis/currentvis.html (accessed on 10 December 2016).

2. Centers for Disease Control and Prevention. Reported Cases and Deaths from Vaccine Preventable Diseases, United States, 1950-2013. In Epidemiology and Prevention of Vaccine-Preventable Diseases, 13th ed.; Hamborsky, J., Kroger, A., Wolfe, S., Eds.; Public Health Foundation: Washington, DC, USA, 2015; pp. E1-E6.

3. Roush, S.W.; Murphy, T.V. Historical comparisons of morbidity and mortality for vaccine-preventable diseases in the United States. J. Am. Med. Assoc. 2007, 298, 2155-2163. [CrossRef] [PubMed]

4. Centers for Disease Control and Prevention. Benefits from Immunization During the Vaccines for Children Program Era-United States, 1994-2013. Morb. Mortal. Wkly. Rep. 2014, 63, 352-355.

5. Zhou, F.; Shefer, A.; Wenger, J.; Messonnier, M.; Wang, L.Y.; Lopez, A.; Moore, M.; Murphy, T.V.; Cortese, M.; Rodewald, L. Economic Evaluation of the Routine Childhood Immunization Program in the United States, 2009. Pediatrics 2014, 133, 577-585. [CrossRef] [PubMed]

6. Centers for Disease Control and Prevention. Rotavirus. In Epidemiology and Prevention of Vaccine-Preventable Diseases, 13th ed.; Hamborsky, J., Kroger, A., Wolfe, S., Eds.; Public Health Foundation: Washington, DC, USA, 2015; pp. 311-324.

7. Simonsen, L.; Reichert, T.A.; Viboud, C.; Blackwelder, W.C.; Taylor, R.J.; Miller, M.A. Impact of influenza vaccination on seasonal mortality in the US elderly population. Arch. Intern Med. 2005, 165, 265-272. [PubMed]

8. Demicheli, V.; Jefferson, T.; Al-Ansary, L.A.; Ferroni, E.; Rivetti, A.; Di Pietrantonj, C. Vaccines for preventing influenza in healthy adults (Review). Cochrane Database Syst. Rev. 2014, 3, 1-2.

9. Jefferson, T. Influenza vaccination: Policy versus evidence. BMJ 2006, 333, 912-915. [CrossRef] [PubMed]

10. Centers for Disease Control and Prevention. Meningococcal Disease. In Epidemiology and Prevention of Vaccine-Preventable Diseases, 13th ed.; Hamborsky, J., Kroger, A., Wolfe, S., Eds.; Public Health Foundation: Washington, DC, USA, 2015; pp. 231-246.

11. Centers for Disease Control and Prevention. Active Bacterial Core Surveillance (ABCs) Report. Emerging Infections Program Network. Streptococcus pneumoniae. 2000. Available online: https://www.cdc.gov/abcs/ reports-findings/survreports/spneu00.pdf (accessed on 22 January 2020).

12. Centers for Disease Control and Prevention. Active Bacterial Core Surveillance (ABCs) Report. Emerging Infections Program Network. Streptococcus pneumoniae. 2009. Available online: https://www.cdc.gov/abcs/ reports-findings/survreports/spneu09.pdf (accessed on 22 January 2020).

13. Centers for Disease Control and Prevention. Wonder Database Search Query for Deaths from Pneumonia 2000-2009. Available online: https://wonder.cdc.gov/ucd-icd10.html (accessed on 22 January 2020).

14. United States Department of Health, Education, and Welfare. Table 1-23-Deaths from 258 Selected Causes, by 5-Year Age Groups, Race, and Sex: United States, 1962. Vital Statistics of the United States 1962 Volume 2-Mortality Part, A; United States Government Printing Office: Washington, DC, USA, 1964; pp. 1-86-1-187.

15. Infoplease Online Encyclopedia. Available online: https://www.infoplease.com/us/births/live-births-andbirth-rates-year (accessed on 21 November 2016).

16. Hamilton, B.E.; Martin, J.A.; Osterman, M.J.K.; Curtin, S.C.; Mathews, T.J. Births: Final Data for 2014. National Vital Statistics Reports; National Center for Health Statistics: Hyattsville, MD, USA, 2015; Volume 64.

17. Centers for Disease Control and Prevention. Measles. In Epidemiology and Prevention of Vaccine-Preventable Diseases, 13th ed.; Hamborsky, J., Kroger, A., Wolfe, S., Eds.; Public Health Foundation: Washington, DC, USA, 2015; pp. 209-230.

18. Perry, R.T.; Halsey, N.A. The clinical significance of measles: A review. J. Infect. Dis. 2004, 189, S4-S16. [PubMed] 
19. Hussey, G.D.; Klein, M. A randomized, controlled trial of vitamin A in children with severe measles. N. Engl. J. Med. 1990, 323, 160-164. [CrossRef] [PubMed]

20. Center for Disease Control. Mumps Surveillance, January 1972-June 1974; CDC: Atlanta, GA, USA, 1974.

21. Centers for Disease Control and Prevention. Rubella. In Epidemiology and Prevention of Vaccine-Preventable Diseases, 13th ed.; Hamborsky, J., Kroger, A., Wolfe, S., Eds.; Public Health Foundation: Washington, DC, USA, 2015; pp. 325-340.

22. National Communicable Disease Center. Rubella Surveillance, June 1969; National Communicable Disease Center: Atlanta, GA, USA, 1969; pp. 1-14.

23. Centers for Disease Control and Prevention. Tetanus. In Epidemiology and Prevention of Vaccine-Preventable Diseases, 13th ed.; Hamborsky, J., Kroger, A., Wolfe, S., Eds.; Public Health Foundation: Washington, DC, USA, 2015; pp. 341-352.

24. Schuman, L.M.; Doull, L.A. Diphtheria Infection and Morbidity in Cleveland, 1937-1939. Am. J. Public Health 1940, 30, 16-24. [CrossRef]

25. United States Department of Health, Education, and Welfare. Figure 18-Death Rates for Diphtheria: Death-Registration States, 1900-32, and United States, 1933-60. Vital Statistics Rates in the United States 1940-1960; U.S. Government Printing Office: Washington, DC, USA, 1968; p. 84.

26. United States Bureau of the Census. Vital Statistics and Health and Medical Care. In Historical Statistics of the United States, Colonial Times to 1970; Bicentennial Edition, Part 1; U.S. Government Printing Office: Washington, DC, USA, 1975; pp. 44-86.

27. Lee, W.W. The Factor of Chance in Diphtheria Mortality. Am. J. Public Health Nation's Health 1929, 19, 855-862. [CrossRef] [PubMed]

28. Centers for Disease Control and Prevention. Diphtheria. In Epidemiology and Prevention of Vaccine-Preventable Diseases, 13th ed.; Hamborsky, J., Kroger, A., Wolfe, S., Eds.; Public Health Foundation: Washington, DC, USA, 2015; pp. 107-118.

29. Clark, C.; Smith, T. The Effects of Ascorbic Acid on Diphtheria Toxin and Intoxicated HeLa Cells. J. Nutr. Sci. Vitaminol. 1976, 22, 313-319. [CrossRef] [PubMed]

30. Schmitt, M.; Holmes, R. Iron-Dependent Regulation of Diphtheria Toxin and Siderophore Expression by the Cloned Corynebacterium diphtheriae Repressor Gene $d t x R$ in C. diphtheriae C7 Strains. Infect. Immun. 1991, 59, 1899-1904. [CrossRef] [PubMed]

31. Collier, R. Diphtheria Toxin: Mode of Action and Structure. Bacteriol. Rev. 1975, 39, 54-85. [CrossRef] [PubMed]

32. Vitek, C.; Wharton, M. Diphtheria in the Former Soviet Union: Reemergence of a Pandemic Disease. Emerg. Infect. Dis. 1998, 4, 539-550. [CrossRef] [PubMed]

33. Centers for Disease Control and Prevention. Pertussis. In Epidemiology and Prevention of Vaccine-Preventable Diseases, 13th ed.; Hamborsky, J., Kroger, A., Wolfe, S., Eds.; Public Health Foundation: Washington, DC, USA, 2015; pp. 261-278.

34. Romanus, V.; Jonsell, R.; Bergquist, S. Pertussis in Sweden after the cessation of general immunization in 1979. Pediatric Infect. Dis. J. 1987, 6, 364-371. [CrossRef] [PubMed]

35. Centers for Disease Control and Prevention. Poliomyelitis. In Epidemiology and Prevention of Vaccine-Preventable Diseases, 11th ed.; Atkinson, W., Wolfe, S., Hamborsky, J., McIntyre, L., Eds.; Public Health Foundation: Washington, DC, USA, 2009; pp. 231-244.

36. Anderson, G.W.; Rondeau, J.L. Absence of Tonsils as a Factor in the Development of Bulbar Poliomyelitis. J. Am. Med Assoc. 1954, 155, 1123-1130. [CrossRef] [PubMed]

37. Horstmann, D.M. Acute poliomyelitis: Relation of physical activity at the time of onset to the course of the disease. J. Am. Med Assoc. 1950, 142, 236-241. [CrossRef] [PubMed]

38. Centers for Disease Control and Prevention. Haemophilus Influenzae Type b. In Epidemiology and Prevention of Vaccine-Preventable Diseases, 13th ed.; Hamborsky, J., Kroger, A., Wolfe, S., Eds.; Public Health Foundation: Washington, DC, USA, 2015; pp. 119-134.

39. Centers for Disease Control and Prevention. Hepatitis B. In Epidemiology and Prevention of Vaccine-Preventable Diseases, 13th ed.; Hamborsky, J., Kroger, A., Wolfe, S., Eds.; Public Health Foundation: Washington, DC, USA, 2015; pp. 149-174. 
40. Centers for Disease Control and Prevention. Wonder Database Search Query for Deaths from Cirrhosis and Liver Cancer 2010-2014. Available online: https://wonder.cdc.gov/ucd-icd10.html. (accessed on 29 October 2016).

41. Meyer, P.A.; Seward, J.F.; Jumaan, A.O.; Wharton, M. Varicella mortality: Trends before vaccine licensure in the United States, 1970-1994. J. Infect. Dis. 2000, 182, 383-390. [CrossRef] [PubMed]

42. Centers for Disease Control and Prevention. Wonder Database Search Query for Population Aged $<1$ Year, 1991-1994. Available online: https://wonder.cdc.gov/Bridged-Race-v2015.HTML (accessed on 29 October 2016).

43. Centers for Disease Control and Prevention. Varicella. In Epidemiology and Prevention of Vaccine-Preventable Diseases, 13th ed.; Hamborsky, J., Kroger, A., Wolfe, S., Eds.; Public Health Foundation: Washington, DC, USA, 2015; pp. 353-376.

44. Centers for Disease Control and Prevention. Vaccine Information Statement: Live Zoster (Shingles) Vaccine ZVL What You Need Know 2018. Available online: https:/www.cdc.gov/vaccines/hcp/vis/vis-statements/ shingles.pdf (accessed on 7 August 2018).

45. Centers for Disease Control and Prevention. Human Papillomavirus. In Epidemiology and Prevention of Vaccine-Preventable Diseases, 13th ed.; Hamborsky, J., Kroger, A., Wolfe, S., Eds.; Public Health Foundation: Washington, DC, USA, 2015; pp. 175-186.

46. Dunne, E.F.; Unger, E.R.; Sternberg, M.; McQuillan, G.; Swan, D.C.; Patel, S.S.; Markowitz, L.E. Prevalence of HPV infection among females in the United States. JAMA 2007, 297, 813-819. [CrossRef] [PubMed]

47. Centers for Disease Control and Prevention. How Many Cancers Are Linked with HPV Each Year? Available online: https://www.cdc.gov/cancer/hpv/statistics/cases.htm. (accessed on 12 April 2017).

48. United States Department of Health, Education, and Welfare. Death Rates for Detailed Causes: Death-Registration States, 1900-1932, and United States, 1933-1960. Vital Statistics Rates in the United States 1940-1960; U.S. Government Printing Office: Washington, DC, USA, 1968; pp. 559-603.

49. Centers for Disease Control and Prevention. CDC Guidance for Evaluating Health-Care Personnel for Hepatitis B Virus Protection and for Administering Postexposure Management. Morb. Mortal. Wkly. Rep. 2013, 62, 1-19.

50. National Cancer Institute. Available online: https://www.cancer.gov/about-cancer/causes-prevention/risk/ infectious-agents/hpv-vaccine-fact-sheet (accessed on 26 May 2017).

(C) 2020 by the authors. Licensee MDPI, Basel, Switzerland. This article is an open access article distributed under the terms and conditions of the Creative Commons Attribution (CC BY) license (http://creativecommons.org/licenses/by/4.0/). 